\title{
Respuesta a medicamentos biológicos en pacientes con artritis reumatoide según nivel de actividad al inicio
}

\author{
Response to biological agents in patients with rheumatoid arthritis according to disease activity \\ state at the beginning of treatment
}

\author{
Javiera Flores ${ }^{1}$, Josefina Durán²
}

\begin{abstract}
Resumen
Introducción: la Ley Ricarte Soto (LRS) permite a pacientes con artritis reumatoide refractaria acceder a medicamentos biológicos. Sin embargo con esta regulación los pacientes inician éstos con actividad alta de enfermedad por un período prolongado, luego de recibir al menos 3 fármacos sintéticos. Previo a la implementación de esta ley no era necesario cumplir estos requisitos. Objetivos: comparar la respuesta a tratamiento lograda con el uso de medicamentos biológicos según niveles de actividad al inicio mediante la comparación de pacientes con biológicos antes y después de la LRS. Métodos: tomando datos del Programa de atención de pacientes con artritis reumatoide de la Red de Salud UC-Christus se compraró grupos de pacientes que accedieron a biológicos pre y post implementación de la LRS. Se analizó el cambio en DAS28 y la categorización de actividad de enfermedad según DAS28. Se realizó una regresión lineal evaluando edad, seropositividad y DAS28 pre tratamiento. Resultados: se encontró una diferencia significativa en el cambio de DAS28 a los 6 meses de tratamiento $(p=0,02)$ y en la regresión solo con el DAS28 pre tratamiento $(p=0,00)$. Dentro del grupo de pacientes que requirió cambio de biológico, los pacientes post ley iniciaron la terapia más activos y presentaban mayor persistencia de actividad severa a los 6 meses de tratamiento (11\% vs 25\%). Conclusiones: si bien el nivel de actividad al inicio no influyó en la respuesta a los 6 meses de tratamiento, si influyó en la persistencia de actividad severa en quienes requirieron cambio de biológico.
\end{abstract}

Palabras clave: artritis reumatoide; inducción de remisión; terapias farmacológicas; agentes antireumáticos; inmunosupresores; medicamentos biológicos

\begin{abstract}
Introduction: Chilean regulations (Ley Ricarte Soto (RS) allow patients with refractory rheumatoid arthritis to have access to biological agents, but because of the requirements of the law, they spend a long period with active disease. Objectives: To compare the effectiveness of treatment with biological agents according to baseline disease activity by comparing subjects initiating biologics previous to and after ley RS. Methods: Using data from the rheumatoid arthritis clinic at Red Salud UC-Christus, we compared groups of patients who had access to biological agents before and after the implementation of the RS law. Change in DAS 28 was analyzed as well as disease activity categories according to DAS 28. We performed linear regression evaluating age, seropositivity, and baseline DAS28. Results: We found a significant difference in the DAS28 score delta at six months of treatment $(p=0.02)$. In linear statistically significant association in the treatment response with the pre-treatment DAS28 $(p=0.00)$, but in the group of patients that required more than one biological agent, the post-LRS group had a higher pre-treatment DAS28 and a higher rate of high disease activity (11\% vs. $25 \%)$ after six months of treatment. Discussion: Although the baseline disease activity level did not influence the final response to treatment, it had an impact on the persistence of severe activity in patients that required more than one biologic agent.
\end{abstract}

Keywords: rheumatoid arthritis; remission induction; drug therapy; antirheumatic agents; immunosuppressive agents; biological agents

Fecha de envío: 7 de abril de 2020 - Fecha de aceptación: 18 de junio de 2020

(1) Escuela de Medicina, Facultad de Medicina, Pontificia Universidad Católica de Chile

(2) Departamento de Reumatología, Facultad de Medicina, Pontificia Universidad Católica de Chile.

Autor de correspondencia: jgduran@uc.cl 


\section{Introducción}

La artritis reumatoide (AR) es una enfermedad inflamatoria crónica sistémica que produce característicamente sinovitis. La prevalencia en Chile es 0,6\% (Durán et al., 2019). Genera una alta carga de enfermedad, estimándose que a los 10 años de evolución un $30 \%$ de los pacientes tendrá discapacidad severa. (Minsal, 2014).

Para disminuir las consecuencias de esta patología es importante el diagnóstico y tratamiento precoz. La Liga Europea contra el Reumatismo (EULAR) y el Colegio Americano de Reumatología (ACR) proponen metotrexato como primera línea (Smolen et al., 2016; Burmester \& Pope, 2017) y usar la estrategia "treat to target" (Smolen et al., 2013; Smolen et al., 2015). Ésta propone que si no se ha alcanzado remisión clínica o actividad leve en un periodo de tiempo determinado se debe aumentar terapia, lo que implica combinar fármacos antireumáticos modificadores de enfermedad (FARMES) sintéticos o añadir biológicos (Smolen et al., 2013). Si los biológicos fallan la recomendación es cambiar a otro biológico, pero no hay evidencia para decidir si debe ser uno que ocupe otro mecanismo de acción (Nam et al., 2017). Pese a que esta estrategia ha demostrado efectividad, $10-20 \%$ de los pacientes no responde adecuadamente, perpetuando la inflamación, el daño articular y sistémico (Minsal, 2016).

En Chile, el 2016 se implementó la Ley Ricarte Soto (LRS) que permite a pacientes con AR refractaria acceder a medicamentos biológicos. Los requisitos para esto son presentar actividad alta a pesar del uso de 3 FARMES, incluyendo metotrexato o leflunomida, en dosis máximas por al menos 6 meses. Sujetos con actividad moderada no pueden acceder a biológicos (Minsal 2019). Esto contrasta con los plazos recomendados en la estrategia "treat to target" en la que si persiste actividad moderada a los 3 meses de terapia se plantea escalar en tratamiento, llegando antes al uso de biológicos en sujetos refractarios.

Siguiendo las guías de la Ley RS algunos sujetos pasarán tiempos prolongados con su enfermedad muy activa, contrario a lo que recomiendan las guías de manejo (Smolen et al, 2016). Previo a la existencia de la LRS, pacientes con isapre tenían la posibilidad de acceder a biológicos según criterio médico y de esta manera se podía solicitar en etapas más precoces y en sujetos menos activos. En este estudio buscamos evaluar la respuesta a tratamiento lograda con el uso de medicamentos biológicos según distintos niveles de actividad al inicio, comparando pacientes que iniciaron biológicos previo a la LRS con aquellos que iniciaron biológicos posterior a la misma.

\section{Materiales y métodos}

Se tomaron datos del policlínico de AR de la Red de Salud UC-CHRISTUS donde se registra cada 3 meses el nivel de actividad de enfermedad, mediante el uso de instrumentos creados con este fin, el DAS28 y HAQ.

Se comparó el grupo con acceso a biológicos previo a la LRS con pacientes que iniciaron biológicos post LRS.

Como medición de respuesta a tratamiento se midió el cambio en DAS28. A su vez se categorizó el DAS28 en remisión con DAS $28<2,6$, actividad leve-moderada con valores $>2,6$ y $<5,1$ y actividad alta con valores mayores a 5,1 (Van Riel \& Renskens, 2016).

Análisis estadístico: Se usó el test T de Student para comparar la variable continua diferencia de DAS28 entre los dos grupos. Se realizó una regresión lineal para esta variable ajustándose por edad, seropositividad y DAS28 previo a tratamiento. Se aplicó test chi² para comparar respuesta en 3 grupos de actividad definida por DAS 28

Se describen los cambios de biológicos como números absolutos al ser pocos casos.

Se utilizó STATA versión 15 para hacer el análisis estadístico.

\section{Resultados}

Se incluyeron 218 pacientes con AR que accedieron a medicamentos biológicos. 63 pacientes los iniciaron antes de la implementación de la LRS y 155 después. La edad promedio para el grupo pre ley fue 57 años y post ley 53. La seropositividad fue $83 \%$ en el grupo pre ley y $87 \%$ en el post ley. Un $58,7 \%$ de los pacientes pre ley iniciaron biológicos con actividad severa versus $94,7 \%$ en el grupo post ley. EI DAS28 promedio en los pacientes pre ley fue 4,94 y en el grupo post ley 6,01 . Con respecto a la respuesta a medicamentos biológicos, luego de 6 meses de tratamiento $31,75 \%$ de los pacientes pre ley logró remisión, 57,14\% alcanzó actividad leve-moderada y $11,11 \%$ mantuvo actividad severa. Dentro de los pacientes post ley 40,6\% logró remisión, 49,62\% actividad leve-moderada y 9,77\% mantuvo actividad severa. El DAS28 post tratamiento promedio en los pacientes pre ley fue 3,21 y en los post ley 3,09. Se utilizó la variable diferencia de DAS28 pre y post tratamiento para comparar los grupos estudiados. El cambio de DAS28 logrado luego de 6 meses de tratamiento fue 1,72 puntos en los pacientes pre ley y 2,89 puntos en los post ley, encontrándose una diferencia significativa ( $p=0,02)$, al ajustarlo por DAS28 pre inicio de biológico, seropositividad y edad. El factor que influye en la diferencia de 
DAS28 lograda es el DAS28 previo inicio de tratamiento $(p=0,00)$. No influyeron la seropositividad $(p=0,91)$ ni la edad $(p=0,059)$. No hubo asociación significativa en la respuesta a tratamiento al iniciar biológicos en distintos niveles de actividad $(p=0,49)$.

Tabla 1: Caracterización de pacientes

\begin{tabular}{l|c|c}
\hline Total de pacientes & \multicolumn{2}{|c}{$\mathbf{2 1 8}$} \\
\hline & Pre LRS $\mathrm{n}=63$ & Post LRS $\mathrm{n}=155$ \\
\hline Edad (años) & 57 & 53 \\
\hline Seropositividad & $87,3 \%$ & $6,01(\sigma 1,07)$ \\
\hline DAS-28 (inicio) & $4,94(\sigma 1,36)$ & $3,09(\sigma 1,35)$ \\
\hline DAS-28 (6 meses) & $3,21(\sigma 1,48)$ & $94,7 \%$ \\
\hline Inicio tratamiento con actividad severa & $58,7 \%$ & $18 \%$ \\
\hline Uso 20 biológico (falta de respuesta) & $17 \%$ & \\
\hline
\end{tabular}

Tabla 2: Categoría final del tratamiento

\begin{tabular}{l|c|c|c}
\hline Total pacientes & \multicolumn{2}{|c|}{$\mathbf{2 1 8}$} & Valor $\mathbf{p}$ \\
\hline & Pre LRS n=63 & Post LRS n=155 & 0,02 \\
\hline Cambio DAS-28 & 1,71 (IC 95\% 1,34-2,10) & $2,98($ IC95\% 2,65-3,13) & 0,49 \\
\hline $\begin{array}{l}\text { Remisión } \\
(6 \text { meses) }\end{array}$ & $31,7 \%$ & $40,6 \%$ & 0,49 \\
\hline $\begin{array}{l}\text { Act. Leve-moderada } \\
6 \text { meses) }\end{array}$ & $57,1 \%$ & $49,6 \%$ & 0,49 \\
\hline $\begin{array}{l}\text { Act. Severa } \\
\text { (6 meses) }\end{array}$ & $11,1 \%$ & $9,7 \%$ & \\
\hline
\end{tabular}

Un $42,8 \%(n=27)$ en el grupo de pacientes pre ley y $18 \%(n=28)$ en el grupo post ley usaron más de un medicamento, cuyos resultados se muestran en la tabla 3. Dos pacientes en el grupo pre ley y cuatro en el grupo post ley no fueron analizados por un registro insuficiente de datos. No se realizó análisis estadístico dado el número reducido de pacientes.

Tabla 3: Características pacientes que requirieron más de 1 biológico

\begin{tabular}{|c|c|c|}
\hline \multirow[t]{2}{*}{ Total pacientes } & \multicolumn{2}{|c|}{218} \\
\hline & Pre LRS $n=63$ & Post LRS $n=155$ \\
\hline \multicolumn{3}{|l|}{ Causas de uso de $>1$ fármaco } \\
\hline No respuesta & $11(40,7 \%)$ & $28(100 \%)$ \\
\hline Costo o preferencia & $10(37,1 \%)$ & 0 \\
\hline RAM o contraindicación & $6(22,2 \%)$ & 0 \\
\hline \multicolumn{3}{|c|}{ Características pacientes con $2^{\circ}$ biológico por no respuesta } \\
\hline Edad (años) & 56 & 54 \\
\hline Seropositividad & $77 \%$ & $89 \%$ \\
\hline DAS-28 inicio & 5,29 & 6,03 \\
\hline $\begin{array}{l}\text { Respuesta a } 2^{\circ} \text { biológico } \\
\text { (remisión/ actividad leve-moderada) }\end{array}$ & $88 \%$ & $75 \%$ \\
\hline
\end{tabular}

\section{Discusión}

En este estudio pudimos comparar la experiencia chilena iniciando biológicos en AR según criterio médico con el inicio de biológicos luego de un período de alta actividad y habiendo recibido mínimo 3 FARMES.
Los datos obtenidos demuestran que previo a la implementación de la ley los pacientes iniciaban biológicos con menor actividad de enfermedad. Los pacientes post ley experimentaron un mayor cambio en su puntaje puesto que iniciaron con un score más alto que los pre-ley, no obstante ambos lograron niveles de actividad 
similares al comparar DAS28 promedio a los 6 meses de tratamiento, con tasas de remisión de $31,7 \%$ en el grupo pre LRS y $40,6 \%$ en el grupo post ley. Estos valores son similares a los reportados en la literatura, donde se describen tasas de remisión entre un 10 y un $60 \%$, debido a la heterogeneidad de los estudios disponibles (Hyrich et al., 2006; Listing et al,. 2006; Schipper et al., 2012; Hmamouchi et al., 2014; Hughes et al., 2018; Hamann et al., 2019). En nuestro estudio el nivel de actividad al inicio del tratamiento no influyó en la respuesta final en cuanto al logro de remisión de la enfermedad.

Dentro de los pacientes que requirieron cambio de biológico, los pacientes post ley tenían DAS28 pre tratamiento más alto que los pre ley, con persistencia de actividad severa mayor que los pre ley ( $25 \%$ vs $11 \%$ ). Las tasas de remisión logradas fueron de $17,8 \%$ en el grupo post ley y $0 \%$ en el pre ley. Se describen tasas de remisión entre un 7,2 y 33 en pacientes que requirieron más de un biológico. (Soliman et al., 2012; Harrold et al., 2015).

Con la implementación de la Ley Ricarte Soto se modifican los criterios de suspensión o cambio de biológico, dado que previo a ella las reacciones adversas a medicamentos y costos fueron una causa importante de suspensión, sin embargo en este estudio se vio que en los casos que accedieron a biológicos posterior a la implementación de la ley la única causa fue la falta de respuesta. Esto demuestra que la LRS está cumpliendo con el objetivo de mejorar el acceso a medicamentos de alto costo en pacientes que lo requieren.

Si bien un porcentaje importante responde satisfactoriamente al tratamiento, el hecho de usar múltiples fármacos implica prolongar los periodos en que el paciente se encuentra con una inflamación importante y aumenta los costos para el sistema de salud.

Una de las limitaciones de este estudio es el riesgo de error tipo Beta (falta de poder). Para un poder de $80 \%$ con valor a de 0,05 , tomando valores de cambio de DAS28 de la literatura, se requería una muestra de 85 pacientes por grupo de comparación, que no se cumplió en el grupo pre LRS. El tamaño muestral de este estudio confiere un poder de $70 \%$. Muchos de los pacientes pre ley no fueron incluidos debido a registro incompleto de datos en las fichas clínicas. En caso de haber estado más activos aquellos pacientes no registrados se genera sesgo de selección. Si bien ambos grupos parecen responder igual, el que los pacientes post LRS tengan que pasar al menos 6 meses con actividad severa para recibir tratamiento tiene repercusiones tales como secuelas articulares, aumento del riesgo cardiovascular, impotencia funcional e incapacidad laboral lo cual afecta la calidad de vida y tiene un costo indirecto en la economía del país. Por otro lado, el score DAS28 tiene la desventaja de ser subjetivo al estar condicionado por la evaluación de cada médico y paciente.

Financiamiento: Departamento de reumatología Pontificia Universidad Católica de Chile.

Contribuciones: Ambos autores participaron en la recopilación de datos, análisis estadístico y redacción del trabajo. Los autores no declaran conflicto de intereses.

\section{Referencias}

Burmester G \& Pope J. (2017). Targeted treatments for rheumatoid arthritis 2. Lancet 389, 2338-2348.

Durán J, Massardo L, Llanos C, Jacobelli S \& Burgos P. et al. (2019). The prevalence of Rheumatoid Arthritis in Chile: a nation-wide study performed as part of the National Health Survey (ENS 201617). J Rheumatol. pii: jrheum.190396.doi:10.3899/jrheum.190396. [Epub ahead of print]

Hamann P, Pauling J, McHugh N, Shaddick G \& Hyrich K, the BSRBR-RA Contributors Group. (2019). Predictors, demographics and frequency of sustained remission and low disease activity in anti-tumour necrosis factor-treated rheumatoid arthritis patients. Rheumatology 58, 2162-2169

Harrold L, Reed G \& Magner R. (2015). Comparative effectiveness and safety of rituximab versus subsequent anti-tumor necrosis factor therapy in patients with rheumatoid arthritis with prior exposure to anti-tumor necrosis factor therapies in the United States Corrona registry. Arthritis Res Ther 17, 256

Hyrich K, Symmons D, Watson K \& Silman A. (2006). Comparison of the response to infliximab or etanercept monotherapy with the response to cotherapy with methotrexate or another disease-modifying antirheumatic drug in patients with rheumatoid arthritis: Results from the British Society for Rheumatology Biologics Register. Arthritis \& Rheumatism 54, 1786-1794.

Hmamouchi I, Combe B, Fautrel B, Rincheval N \& Lukas C. (2014). Prevalence and concordance of sustained remission assessed by various validated indices in the early arthritis "ESPOIR" cohort. Joint Bone Spine 81, 409-415.

Hughes C, Scott D \& Ibrahim F. (2018). Intensive therapy and remissions in rheumatoid arthritis: a systematic review. BMC Musculoskelet Disord 19, 389 
Listing J, Strangfeld A \& Rau R (2006). Clinical and functional remission: even though biologics are superior to conventional DMARDs overall success rates remain low - results from RABBIT, the German biologics register. Arthritis Res Ther 8, R66

Minsal. (2014). Guía clínica Artritis Reumatoide, Ministerio de Salud. Gobierno de Chile, 12-14. Accedido en: https://www.minsal.cl/sites/ default/files/files/GPC\%20Artritis.pdf el 31 marzo 2020

Minsal. (2016). Tratamiento con medicamentos biológicos, Etanecept o Abatacept o Adalimumab o Rituximab en adultos con Artritis Reumatoide refractaria a tratamiento habitual. Ministerio de Salud. Gobierno de Chile, 9-10. Accedido en: https://www.minsal.cl/ wp-content/uploads/2015/08/6_Protocolo-AR.pdf el 31 marzo 2020.

Minsal. (2018). Tratamiento con Etanercept o Abatacept o Adalimumab o Rituximab en personas con Artritis Reumatoide activa refractaria a tratamiento habitual. Ministerio de Salud. Gobierno de Chile, 8-11. Accedido en: https://www.minsal.cl/wp-content/uploads/2018/03/ Protocolo-Artritis-Reumatoide.pdf el 31 marzo 2020.

Nam J, TakaseMinegishi K, Ramiro S., et al. (2017). Efficacy of biological disease-modifying antirheumatic drugs: a systematic literature review informing the 2016 update of the EULAR recommendations for the management of rheumatoid arthritis. AnnRheumDis 76, 1108-1113.

Schipper L, Vermeer M \& Kuper H. (2012). A tight control treatment strategy aiming for remission in early rheumatoid arthritis is more effective than usual care treatment in daily clinical practice: a study of two cohorts in the Dutch Rheumatoid Arthritis Monitoring registry. AnnRheumDis 71, 845-850
Smolen J, Aletaha D \& Mclnenes I. (2016). Rheumatoid arthritis. Lancet 388, 2023-38.

Smolen J, Landewe R \& Breedveld F. (2014). EULAR recomendentions for the managment of rheumatoid arthritis with synthetis and biological disease-modifying antirheumatic drugs: 2013 update. AnnRheumDis 73, 492-509

Smolen J, Landewe R \& Breedveld F. (2015). Treating rheumatoid arthritis to target: 2014 update of the recommendations of an international task force. AnnRheumDis 0, 1-13

Smolen JS, Landewé R Bijlsma J., et al.(2017). EULAR recommendations for the management of rheumatoid arthritis with synthetic and biological disease-modifying antirheumatic drugs: 2016 update AnnRheumDis 76, 960-977.

Soliman M, Hyrich K, Lunt M, Watson K, Symmons D \& Ashcroft D on Behalf of the british society for rheumatology biologics register. (2012). Rituximab or a Second Anti-Tumor Necrosis Factor Therapy for Rheumatoid Arthritis Patients Who Have Failed Their First Anti-Tumor Necrosis Factor Therapy? Comparative Analysis From the British Society for Rheumatology Biologics Register. Arthritis Care \& Research 64, $1108-1115$.

Van Riel PL \& Renskers L. (2016). The Disease Activity Score (DAS) and the Disease Activity Score using 28 joints counts (DAS28) in the management of rheumatoid arthritis. Clin Exp Rheumatol 34, S40-44 